\title{
Insight to Pyroptosis in Viral Infectious Diseases
}

\author{
Junxia Li, Lijun Chen, Changhui Zhou, Yifan Bai, Ruiyan Zhao, \\ Jinge Zhang, Xiaoqiao Xu, Xingyi Ge*, Ye Qiu* \\ College of Biology, Hunan University, Changsha, China \\ Email: *xyge@hnu.edu.cn, ${ }^{*}$ qiuye@hnu.edu.cn
}

How to cite this paper: Li, J.X., Chen, L.J., Zhou, C.H., Bai, Y.F., Zhao, R.Y., Zhang, J.G., Xu, X.Q., Ge, X.Y. and Qiu, Y. (2021) Insight to Pyroptosis in Viral Infectious Diseases. Health, 13, 574-590.

https://doi.org/10.4236/health.2021.135043

Received: April 15, 2021

Accepted: May 21, 2021

Published: May 24, 2021

Copyright $\odot 2021$ by author(s) and Scientific Research Publishing Inc. This work is licensed under the Creative Commons Attribution-NonCommercial International License (CC BY-NC 4.0). http://creativecommons.org/licenses/by-nc/4.0/ (c) (i) (8) Open Access

\begin{abstract}
Background: Pyroptosis is defined as programmed necrosis executed by gasdermin D or E (GSDMD or GSDME), which punches cellular membrane. Morphologically, pyroptosis is characterized by cell swelling and cell membrane rupture, leading to the release of cellular contents that triggers intense inflammatory response. More and more studies have found that pyroptosis may be involved in the pathogenesis of viral infection, which may be a determinant for inflammation observed in most viral diseases. Objective: This paper aims to summarize the roles of pyroptosis in the pathogenesis of viral infectious diseases and to provide potential drug targets for the treatment of viral diseases, which will contribute to medical research and public health. Measures: This paper mainly summarizes pyroptosis occurring in diseases caused by different viruses, including human immunodeficiency virus, hepatitis virus, enterovirus, influenza virus and dengue fever virus. Meanwhile, the reported mechanism underlying pyroptosis mediating pathogenesis of these viral diseases will also be described. Conclusion: Current studies have shown that pyroptosis is a double-edged sword in viral infectious diseases. On one hand, pyroptosis leads to pathogenic inflammation of many viral infectious diseases which aggravate tissue damage initiated by viral infection, and blocking proptosis usually relieves the inflammation, which exerts therapeutic effects on viral diseases. On the other hand, moderating pyroptosis can contribute to defense against pathogen infection by releasing immune epitopes and inducing antiviral immune response.
\end{abstract}

\section{Keywords}

Pyroptosis, Viral Infectious Diseases, Gasdermin D, Gasdermin E, Caspase, Inflammation 


\section{Background and Purpose}

Cell death modes such as necrosis, autophagy and apoptosis have been gradually discovered. In recent years, a new type of cell death, pyroptosis, has been described. Pyroptosis is a kind of programmed and inflammatory necrosis, depending on caspase (cysteinyl aspartate specific proteinase) activation and the cell-membrane-pore forming executed by the protein family of gasdermins. Pyroptosis results in lytic cell death accompanied by the release of inflammatory factors, inducing cascade and amplification of inflammatory response. Pyroptosis is an important immune defense mechanism in the body, which plays an important role in resisting invasion of external pathogens and sensing internal pathogenic signals within cells.

Along with the whole human history, from epidemics recorded on murals of ancient Egypt to the recent global COVID-19 pandemic, viral infectious diseases have been imposing great threat to human health. Inflammation is a defensive response against viral infection in the body, but exaggerated inflammation will lead to tissue damage and exacerbated the disease. As a process of inflammatory cell death, pyroptosis has been reported to be closely related to inflammatory modulation in various viral infections. Thus, better understanding of pyroptosis will provide new ideas for the prevention and treatment of viral infectious diseases. In this paper, we summarize some studies about the relationship between pyroptosis and viral infectious diseases that have been discovered at present, focusing on a small but in-depth scope, which can provide a practical reference value for researchers studying viral infectious diseases and pyroptosis.

\section{Morphological Features of Pyroptosis}

Pyroptosis was first described by Zychlinsky and his colleagues in 1992 when they observed that Shigella fredori infected macrophages and underwent lytic cell death which shares some characteristics with apoptosis, such as DNA fragmentation, nuclear pyknosis and caspase-dependence, and thus was thought to be apoptosis originally [1]. In 2001, Cookson et al. found that though such lytic cell death mode depends on caspases, it shows some features different from caspase-3-mediated apoptosis, including the formation of pores on the cell membrane, cell lysis and release of contents, and thus renamed it as pyroptosis. "Pyro" means fire, indicating that this programmed cell death can trigger an inflammatory response, and "ptosis" means falling, representing the nature of programmed cell death [2]. The original pattern of pyroptosis is caspase-1-dependent in which gasdermin $\mathrm{D}$, a perforating protein, is cleaved and activated by activated caspase 1 , and the N-terminal cleavage band can recognize and bind to the cell membrane, assembling into hollow annular pores with a diameter of $10-15 \mathrm{~nm}$ [3]. Because the balance of the membrane permeation barrier is destroyed by the perforating, $\mathrm{K}^{+}$outflows and $\mathrm{Na}^{+}$inflows, accompanied by the inflow of other extracellular solution, leading to the expansion of cells [4]. Morphologically, pyroptotic cells blow out many "bubbles" on their surface at first. After a certain 
time, the bubbles burst, and the cell contents including interleukin $1 \beta$ (IL-1 $\beta$ ), IL-18 and lactate dehydrogenase are released to the extracellular fluid through the pore channels, which will recruit immune cells and trigger a wide range of inflammatory reactions [5]-[11].

\section{The Molecular Mechanism of Pyroptosis}

In the process of pyroptosis, intracellular inflammatory bodies form specific protein complexes firstly in response to the stimulation of different signals, leading to the activation of different caspases which cleave gasdermin $\mathrm{D}$ or $\mathrm{E}$ to release N-terminal fragments that form pores on the cell membrane, resulting in cell membrane rupture and release of cell contents. Pyroptosis causes a strong inflammatory response. According to the dependence on the inflammatory caspase, pyroptosis can be classified into canonical and non-canonical pathways. The mechanism of pyroptosis is summarized in Figure 1.

\subsection{Overview of Caspase Family Proteins}

Caspases are a group of proteases with similar structure existing in the cytoplasm. In 1993, Yuan et al. found that the CED-3 gene of C. elegans is highly homologous in function and sequence to the ICE (Interleukin-18 converting enzyme) gene of mammalian cells, which is similar to the CED-3 gene. The high expression of ICE gene can induce the apoptosis of rodent fibroblasts, attracting much attention. More and more researchers have carried out a series of studies on the relationship between caspase family proteins and apoptosis. All caspases

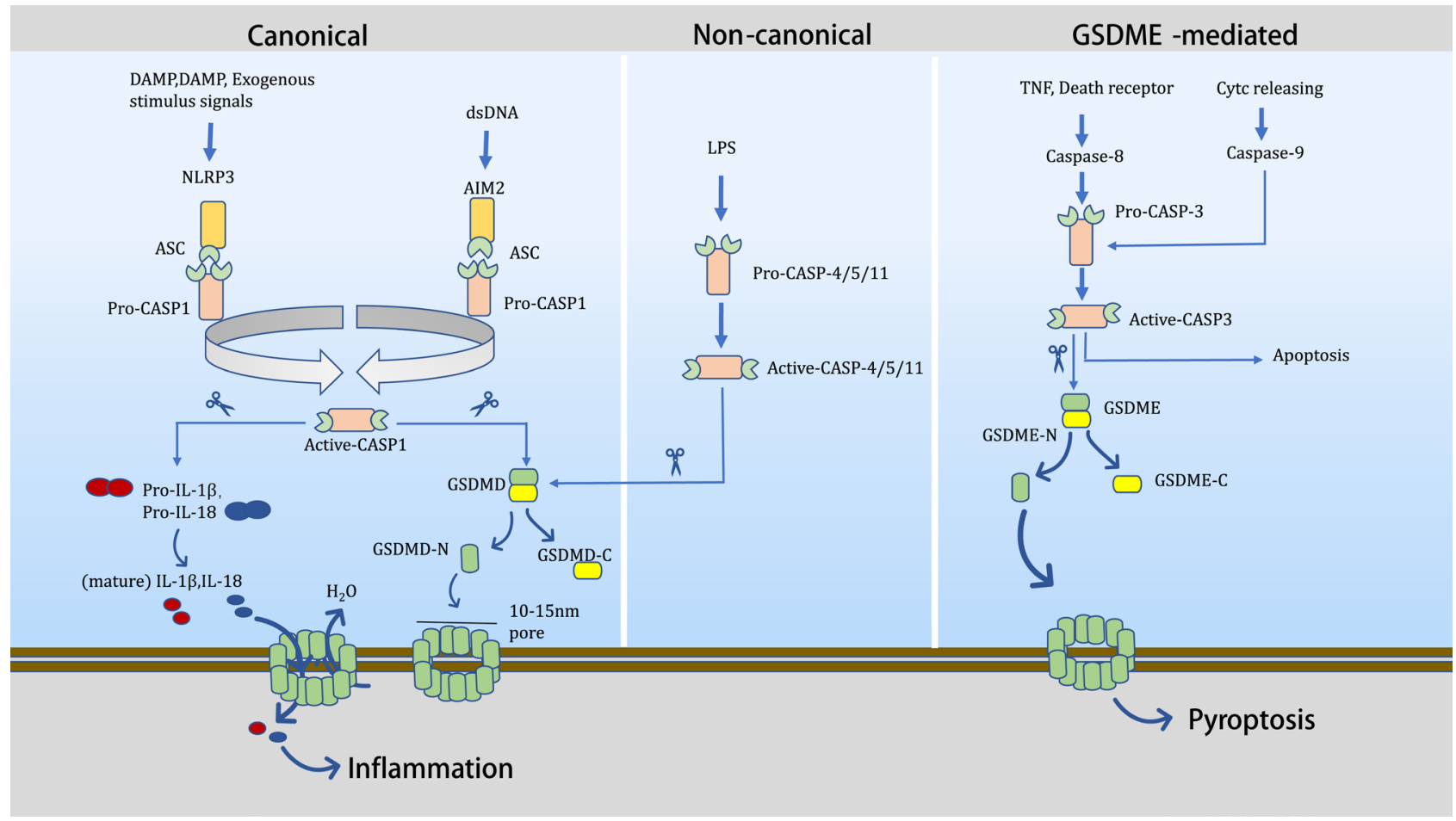

Figure 1. Mechanisms and pathways of pyroptosis. 
are characterized by the conserved pentapeptide sequence of OACXG (X being any amino acid) and their similar functions. At least 14 members of the caspase family have been found in mammals, named caspase 1 to caspase 14 in sequence according to the time of discovery [12]. The inflammatory factors related to pyroptosis include caspase 1 , caspase 4 , caspase 5 , caspase 11 , caspase 12 , caspase 13 and caspase 14 [13]. The expression of caspases is species dependent. For instance, mouse mainly expresses caspase 1 and caspase 11, but human mainly expresses caspase 1, caspase 4 and caspase 5 [14].

Caspase family proteins share similar amino acid sequence, structure and substrate specificity. In live cells, they generally exist in the form of inactive proteinogen which can be activated through proteolysis. The prozyme molecule is composed of three parts, including an $\mathrm{N}$-terminal primordial domain, a large subumit and a small subunit. Upon activated, the large subunit and the small subunit form a heterodimer, and the two heterodimers will assemble into an enzymatic active heterotetramer. Previous studies have confirmed that the caspase family proteins are related to a variety of physiological and pathological processes. For example, caspase 12 can inhibit the activation of caspase 1 and further inhibit the occurrence of inflammatory response. Cancer rates are higher after loss of caspase 2; Caspase 3 is associated with GSDME-mediated pyroptosis and apoptosis signaling pathways. Caspase 6, 7, 8, 9 and 10 are all involved in apoptosis, among which caspase 9 is closely related to the generation of degenerative diseases and cancer [13] [15] [16] [17] [18].

\subsection{Overview of Gasdermin Family Proteins}

The gasdermin protein family consists of six members, they are gasdermin A (GSDMA), gasdermin B (GSDMB), gasdermin C (GSDMC), gasdermin D (GSDMD) and gasdermin E (GSDME/DFNA5) and an autosomal recessive genetic deafness protein No. 59 (Deafness, Autosomal Recessive 59, DFNB59) [19] [20]. Each member of the gasdermin family has a different distribution and function in human tissues and organs.

GSDMA is mainly found in the epithelial cells of the skin, esophagus, tongue and umbilical cord, and is associated with the development of diabetes and asthma, as well as a number of other immunological diseases.

GSDMB is highly expressed in liver, stomach, skin and immune cells, and is associated with diabetes, inflammatory bowel disease, colitis and asthma [21] [22].

GSDMC is mainly localized in tissues such as skin, spleen, esophagia and bladder, and was initially thought to be the exonuclear factor of the melanoma-derived leucine zipper due to its dominant expression in melanoma cells [9].

The GSDMD gene is located at 8q24 and is mainly expressed in skin, immune cells and gastrointestinal tissues. It is composed of 242 amino acids and can be divided into three parts: The $\mathrm{N}$-terminal region, the intermediate junction region and the C-terminal region [23] [24]. By far, GSDMD is the most common and well-studied pyroptosis associated protein. In GSDMD mediated pyroptosis, 
GSDMD can be cleaved by caspase1/4/5/11 to release C-terminal fragment and $\mathrm{N}$-terminal fragment with pyroptosis effector. The N-terminal fragment is lipophilic and can interact with PI (Phosphatidylinositol), PS (Phosphatidylserine) on the inner side of the cell membrane, and Cardiolipin on both sides of the bacterial membrane specifically bind and assemble to form pores, which disrupt the balance of the membrane permeability barrier, resulting in $\mathrm{K}+$ outflow, $\mathrm{Na}+$ inflow, and water molecules from the outside. The cells swell, their membranes rupture, and their contents are released, leading to pyroptosis and an intense inflammatory response. Studies have shown that when the N-terminal domain forms fewer channels, cells can initiate compensatory repair mechanism to repair the damage of cell membrane. However, when the $\mathrm{N}$ terminal of GSDMD makes too many holes in the cell, it will exceed the repair ability of the cell, which will lead to cell lysis and release of cell contents, causing pyroptosis [5] [7] [9] [10] [11].

GSDMD can combine with the cardiolipin on the bacterial cell membrane and punch holes to play a bactericidal role, which can effectively kill Staphylococcus aureus, Escherichia coli and Bacillus etc. [25]. In addition, it has been found that the C-terminal fragment of GSDMD inhibits pyroptosis induced by $\mathrm{N}$-terminal fragment. Under normal physiological conditions, the GSDMD-C-terminal domain inhibits the drilling activity by connecting with the GSDMD-N-terminal domain, so as to maintain the stability of the internal environment [26] [27].

GSDME is the earliest gasdermin family protein discovered, and it is the second protein capable of mediating pyroptosis following GSDMD. Because its mutation is related to the occurrence of hereditary deafness, it was originally named DFNA5. It is mainly expressed in cochlea, intestinal tract, placenta and brain. In recent years, GSDME-related pyroptosis has drawn more and more attention [28]. Studies have shown that in the treatment of chemotherapy drugs, GSDME can be cleaved by caspase 3 between the 275th and 276th amino acid residues, releasing about $33 \mathrm{kDa}$ GSDME-N fragment which has a function of perforating the cell membrane. This molecular mechanism is similar to that of GSDMD mediated pyroptosis [29] [30].

GSDME-mediated pyroptosis is one of the reasons for the side effects of chemotherapeutic drugs that damages normal tissues, since GSDME is expressed at a high level in many healthy tissues. Recent studies have shown that when caspase 3 is activated, the expression level of intracellular GSDME determines the mode of cell death. When the expression level of intracellular GSDME is high, activated caspase 3 cleaves GSDME and induces pyroptosis. When the expression level of GSDME is low or there is no GSDME expression, the cells undergo apoptosis [30]. Therefore, GSDME is a key molecule switching capase-3-mediated apoptosis and pyroptosis.

\subsection{The Canonical Pyroptosis Pathway}

When the body encounters a variety of infectious and immunological stimuli, 
caspase 1 can be activated through different inflammatory bodies, which initiates the canonical pyroptosis pathway. When pathogens invade, inflammasomes can recruit caspase 1 precursors directly or through ASC (apoptosis-associated speck-like protein containing C-terminal caspase recruitment domain), resulting in hydrolysis of caspase 1 precursors and production of active caspase 1. The inflammasomes containing ASC can interact with the caspase 1 homologous protein PYD (pyrin domain) to recruit the upstream and downstream PRRs (pattern recognition receptors) and the active form of caspase 1, assembling inflammatory complex [31] [32]. As for some inflammasomes lacking ASC connectors, such as NAIP-NLRC4 and NLRP1b inflammasomes, they directly recruit pro-caspase 1 and activate it, but do not produce inflammatory complex [33]. Further studies have revealed the specific molecular mechanism of GSDMD-induced pyroptosis. Accurate structural analysis results showed that when the signal was transmitted to GSDMD by the mature caspase 1 in the cell, GSDMD will be cleaved into the lipophilic fragment of the GSDMD-N terminus of $31 \mathrm{kDa}$ and the hydrophilic fragment of the GSDMD-C terminus of $22 \mathrm{kDa}$ [34]. GSDME-N can specifically bind to phosphatidylinositol (PI) and phosphatidylserine (PS) on the inner side of cell membrane and assemble pores. Under electron microscopy, there are about $16 \mathrm{~N}$-terminal segments assembling hollow annular pores with a diameter of $10-15 \mathrm{~nm}$. On the other hand, activated caspase 1 can also cleavage the inflammatory cytokine IL- $1 \beta$ and IL-18 precursors to generate mature IL- $1 \beta$ and IL-18. As pore-forming destroys membrane-permeability barrier, $\mathrm{K}^{+}$outflows and $\mathrm{Na}^{+}$inflows together with other extracellular solutes, leading to cell expansion and cell membrane rupture. Mature inflammatory cytokines IL- $1 \beta$ and IL-18 can also be released to extracellular fluid through the pore, which in turn stimulates the recruitment of immune cells and triggers a cascade of inflammatory responses [5] [7] [9] [10] [11].

\subsection{Non-Canonical Pyroptosis Pathway}

Human caspase $4 / 5$ and murine caspase 11 can be activated by Toll-like receptor combined with lipopolysaccharide (LPS) of gram-negative bacteria. Caspase 4/5/11 directly binds to lipid A, the conserved domain of LPS, for its activation. The activated caspase 4/5/11 then cleaves GSDMD, and the GSDMD-N fragment is generated to perform the function of cell perforation, thus leading to inflammatory necrosis. This pathway, which is dependent on caspase $4 / 5 / 11$, is known as the non-canonical pathway. Caspase 11 is also an upstream activator of caspase 1 that activates caspase 1 together with NLRP3 and ASC, further triggering pyroptosis and releasing mature IL-1 $\beta$ and IL-18 [35].

\subsection{GSDME-Mediated Pyroptosis Pathway}

Activation of caspase 3 was generally believed as a marker of apoptosis. For example, in the treatment of cancer with chemotherapy drugs such as doxorubicin and cisplatin, caspase 8 and caspase 9 can be activated, and then activate down- 
stream caspase 3 to induce exogenous and endogenous apoptosis, respectively [36] [37]. But recent studies have shown that activated caspase 3 also cleaves GSDME to release active GSDME-N terminal fragment which can also drill into the cell membrane. Similar to GSDMD, GSDME-mediated pyroptosis also causes cell swell and rupture, releasing some inflammatory cytokines and other cellular contents, recruiting extracellular immune cells, and triggering a strong inflammatory cascade reaction [29] [30].

GSDME has been found to be expressed at low levels in many cancer cells, but at high levels in normal tissues, such as the placenta, kidney, lung, uterus and heart. When Shao Feng et al. studied the association between chemotherapy drugs treatment and GSDME mediated cell apoptosis, they found that damage of tissue and organ in wild-type mice treated with chemotherapy drugs was significantly more serious than that in gsdme $^{-/-}$mice, indicating that in the process of cancer treatment, the usage of chemotherapy drugs can cause serious side effects on the patient's normal tissues and organs. Therefore, the discovery of caspase 3-GSDME mediated pyroptosis pathway can provide new ideas and new targets for the treatment of cancer and the prevention of side effects caused by chemotherapy drug [30].

\section{Role of Pyroptosis in Viral Infectious Diseases}

Table 1. Role of pyroptosis in viral infectious diseases.

\begin{tabular}{|c|c|c|c|}
\hline Virus & Disease & $\begin{array}{c}\text { Role of pyroptosis in virus-induced } \\
\text { disease }\end{array}$ & References \\
\hline SARS-CoV-2 & $\begin{array}{l}\text { COVID-19, Pneumonia, } \\
\text { Severe acute Respiratory } \\
\text { syndrome, Kidney failure. }\end{array}$ & $\begin{array}{l}\text { Hematopoietic stem cell pyroptosis. } \\
\text { Levels of IL- } 1 \beta \text { and LDH were } \\
\text { significantly increased in COVID-19 } \\
\text { patients. }\end{array}$ & {$[38]-[49]$} \\
\hline $\begin{array}{l}\text { Hepatitis } \\
\text { virus }\end{array}$ & $\begin{array}{l}\text { Viral hepatitis, Liver fibrosis, } \\
\text { Cirrhosis of the liver, Hepatic } \\
\text { carcinoma. }\end{array}$ & $\begin{array}{c}\text { HBeAg inhibits pyroptosis. } \\
\text { HCV causes pyroptosis. } \\
\text { Inhibition of NLRP3 relieves acute liver } \\
\text { failure induced by viral hepatitis and } \\
\text { inhibit pyroptosis. }\end{array}$ & $\begin{array}{l}{[57][58]} \\
{[59]} \\
{[60][61]}\end{array}$ \\
\hline $\begin{array}{l}\text { Influenza } \\
\text { virus }\end{array}$ & Influenza & $\begin{array}{l}\text { H5N1 acts on galectin-3 and activates } \\
\text { NLRP3, promoting pyroptosis in the } \\
\text { lungs and triggering inflammation. }\end{array}$ & $\begin{array}{l}{[50][51]} \\
\quad[52]\end{array}$ \\
\hline HIV & AIDS & $\begin{array}{l}\text { HIV destroys immune system by } \\
\text { causing pyroptosis of } \mathrm{CD} 4^{+} \mathrm{T} \text { cells. }\end{array}$ & $\begin{array}{l}{[53][54]} \\
{[55][56]}\end{array}$ \\
\hline DENV & $\begin{array}{l}\text { Dengue haemorrhagic fever, } \\
\text { Dengue shock syndrome. }\end{array}$ & $\begin{array}{c}\text { Dengue virus causes pyroptosis of } \\
\text { monocytes and macrophage. }\end{array}$ & {$[62][63]$} \\
\hline Enterovirus & $\begin{array}{c}\text { HFMD, Myocarditis, } \\
\text { Pancreatitis, Meningitis }\end{array}$ & $\begin{array}{l}\text { EV71 and CVB3 induce pyroptosis via } \\
\text { caspase 1-GSDMD pathway. }\end{array}$ & [64] [65] \\
\hline Other viruses & Hydrophobia, Enterogastritis & $\begin{array}{l}\text { Rabies virus activates pyroptosis via } \\
\text { caspase } 1-\text { GSDMD pathway. }\end{array}$ & {$[66][67]$} \\
\hline
\end{tabular}

\subsection{Role of Pyroptosis in Novel Coronavirus Infection}

Novel coronavirus (SARS-CoV-2) is a novel coronavirus discovered in Decem- 
ber 2019 which causes global pandemic of COVID-19 and greatly threats public health. Currently, its spread is still raging around the world [38] [39]. Studies have reported that SARS-CoV-2 infection can induce a strong inflammatory cytokine storm [40] [41], resulting in loss of hematopoietic function and lymphocytopenia in the body [42] [43]. In recent years, it has been found that pyroptosis can also lead to a strong inflammatory response, and it can clear or reduce the number of cells by cleavage, which is exactly in line with the symptoms after SARS-CoV-2 infection. There is growing evidence indicating that pyroptosis may be involved in the infection and pathogenesis of SARS-CoV-2. NLRP3 inflammasome is likely to be the culprit for certain complications of SARS-CoV-2 infection that may affect multiple tissues and organs as well as potential hematopoietic function [41] [44].

During infection, SARS-CoV-2 spines can recognize ACE2, its receptor, on the cell surface and directly activate NLRP3 inflammasome. NLRP3 inflammasome can subsequently activate caspase 1 to trigger inflammatory immune response via the release of mature pro-inflammatory factors such as IL-1 $\beta$ and IL-18, triggering a strong inflammatory response. Caspase 1 can also directly lyse GSDMD to produce the active fragment of GSDMD-N, leading to pyroptosis [45] [46] [47]. Many evidences have been demonstrated to support the occurrence of pyroptosis in SARS-CoV-2 infection. Yhan et al. found that the level of lactate dehydrogenase (LDH) in samples from COVID-19 patients was significantly increased [48]. LDH is a cytosolutase residing in the cytoplasm. LDH can only be released into the extracellular environment when the cell membrane is ruptured. Therefore, $\mathrm{LDH}$ can be used as one of the indicators to monitor pyroptosis. In addition, the inflammatory cytokine IL- $1 \beta$, one of the downstream indicators of pyroptosis, is significantly increased in serum of COVID-19 patients [49]. However, the specific pattern of pyroptosis involved in the infection and pathogenicity of SARS-CoV-2 remains to be clarified. Therefore, it is necessary to conduct more in-depth studies on the role of pyroptosis in SARS-CoV-2 infection, in order to provide new ideas for the development of drugs and treatment regimens for COVID-19 (Table 1, Line 1).

\subsection{Role of Pyroptosis in Influenza Virus Infection}

Influenza viruses mutate quickly and have a high rate of gene recombination, so they can cause a massive epidemic and spread after infection, which is one of the reasons for the limited development of influenza vaccine [50]. Influenza virus infection causes an inflammatory response that can lead to severe sequelae, other complications and even death, the study reported. NLRP3 inflammasome is the main antiviral defense mechanism in the host during influenza virus infection. Previous studies have shown that NLRP3 inflammasome activates caspase 1, which leads to the release of mature IL- $1 \beta$ and IL-18 as well as pyroptosis.

Galectin-3 is a $\beta$-galactose lateral binding protein that is widely distributed in immune cells and epithelial cells and can regulate the occurrence of a variety of 
immune processes and microbial infection. Chen et al. found that galectin-3 could promote the activation of NLRP3 inflammasome and enhance the lung inflammation induced by $\mathrm{H} 5 \mathrm{~N} 1$ avian influenza virus in the lung tissue of galectin-3 knockout mice and wild-type mice infected with $\mathrm{H} 5 \mathrm{~N} 1$ influenza virus [51]. In addition, Kuriakose et al. found that during the infection of influenza $A$ virus (IAV), Z-DNA-binding protein 1 can activate NLRP3 inflammasome, and induce necrosis, apoptosis and pyroptosis [52]. Therefore, the inhibition of pyroptosis may provide a new preventive measure for the containment of influenza virus pandemic (Table 1, Line 3 ).

\subsection{Role of Pyroptosis in Human Immunodeficiency Virus Infection}

Human immunodeficiency virus (HIV) infection will cause acquired immune deficiency syndrome (AIDS). HIV can be divided into two related but different groups: The human immunodeficiency virus type-1 (HIV-1) and the human immunodeficiency virus type-2 (HIV-2) [53]. Studies have reported that the main pathogen of AIDS is HIV-1 which mainly attacked CD4 ${ }^{+}$cells including T4 lymphocytes, monocytes macrophages and dendritic cells, resulting in a gradual decline in the number of $\mathrm{CD}^{+} \mathrm{T}$ cells, lymphocyte depletion and paralysis of the host immune system [54]. Due to the correlation between apoptosis and the occurrence and progression of AIDS, previous studies believed that the death and sharp decrease of $\mathrm{CD} 4^{+} \mathrm{T}$ cells were mainly caused by apoptosis [54].

Doitsh et al. examined the spleen and tonsil tissues of HIV-1 infected people and found that only $5 \%$ of $\mathrm{CD}^{+} \mathrm{T}$ cells were infected with HIV-1 and underwent apoptosis [55] [56]. About $95 \%$ of $\mathrm{CD}^{+} \mathrm{T}$ cells were not infected with HIV-1 but died. Further investigation found that caspase 1 was activated in the $95 \%$ cells, and IL- $1 \beta$ and IL- 18 secreted by the cells were significantly increased, suggesting that most of the death of $\mathrm{CD} 4^{+} \mathrm{T}$ cells was caused by pyroptosis mediated by caspase 1 . Subsequent studies using VX-765, a caspase 1 inhibitor, showed that inhibition of caspase 1 cleavage reduces the secretion of IL- $1 \beta$, as well as the death of HIV-infected $\mathrm{CD}^{+} \mathrm{T}$ cells. These results suggest that pyroptosis is involved in the molecular mechanism of the depletion of human immune cells after HIV infection [55] (Table 1, Line 4).

\subsection{Role of Pyroptosis in Hepatitis Virus Infection}

Human hepatitis viruses can be classified into type A, B, C, D, E and G. Hepatitis virus infection can lead to a series of liver damages such as liver fibrosis, cirrhosis of the liver and even hepatic carcinoma. However, the molecular mechanism of liver damage caused by hepatitis virus has not yet been fully confirmed.

Kupffer cells (KCs) are common non-parenchymal cells in the liver, which have a momentous position in maintaining the homeostasis and are main source of inflammasome in the liver [57]. Studies have shown that after HBV invades the host, it can infect the KCS of the host liver, which then responds by releasing 
inflammatory cytokines (such as IL-18) and stimulating natural killer cells (NK) [58]. In the infection of hepatitis B virus (HBV), HBeAg, a component of the core antigen of $\mathrm{HBV}$, can inhibit the activation of caspase 1 and the maturation and releasing of IL- $1 \beta$ by suppressing the production of reactive oxygen species, which is beneficial to the persistent reproduction and immune tolerance of HBV [59]. Kofahi et al. tested the effect of HCV infection on programmed cell death of Huh-7.5 and found that the proliferation rate of HCV infected cells was significantly reduced, accompanied by caspase-3-mediated pyroptosis, and HCV resulted in a significant increase in the proportion of activated caspase 1 [60]. In addition, Wang et al. used a mouse model of hepatic failure that was pathologically similar to viral hepatitis and found that the levels of detected inflammatory cytokines were remarkably reduced after NLRP3 inflammasomes were treated with pyroptosis inhibitors [61]. These results suggest that pyroptosis is a vital mode of cell death in cells infected by hepatitis viruses. Therefore, an in-depth study on the role of pyroptosis in the pathogenesis of hepatitis virus and the way that hepatitis virus uses pyroptosis to evade the immune defense mechanism of the body may provide a new idea for the research and treatment of viral hepatitis (Table 1, Line 2).

\subsection{Role of Pyroptosis in Dengue Virus Infection}

Dengue is a mild and self-limited disease caused by Dengue virus (DENV) infection. A very rare group of patients may develop a worsening condition leading to a more serious and lethal Dengue haemorrhagic fever or Dengue shock syndrome. However, the pathogenic mechanism of Dengue related illness is not clear yet. Tan et al. examined the activation of caspase 1 precursors in primary monocytes infected with DENV and found that pyroptosis is involved in the activation of caspase 1 in DENV-infected monocytes and may play a pro-inflammatory role in the immune pathogenesis of dengue fever [62]. Wu et al. detected that the IL-1 $\beta$ precursor, IL-18 precursor and NLRP3 related to caspase 1 activation were up-regulated in DENV-infected human monocyte derived macrophages, and then they inhibited the NLRP3 inflammasome in macrophages, and found that the pyroptosis phenomenon was significantly reduced [63] (Table 1, Line 5).

\subsection{Role of Pyroptosis in Enterovirus Infection}

Enterovirus 71 (EV71), one of the main causes of hand, foot and mouth disease (HFMD), also affects the nervous system and causes inflammation in patients. Pyroptosis is a newly discovered way of programmed cell lysis and inflammatory necrosis. Since EV71 can also trigger inflammatory response, we speculate that pyroptosis has some connection to the pathogenesis of EV71. In 2017, Zhong et al. found that EV71 infection could induce the activation of caspase 1 and increased the secretion of IL-1 $\beta$ and IL-18 in infected cells [64]. However, when caspase 1 was treated with specific inhibitors, it was discovered that the degree of body damage and inflammation caused by EV71 infection in mice was remarka- 
bly decreased. In addition, they found that caspase 1 inhibitors inhibited EV71 replication in the mouse brain. Similarly, they detected that infection with coxsackie B3 (CVB3), an important member of the enterovirus family, also activated pyroptosis. In HeLa cells infected with CVB3, caspase 1 is activated, and the expression of IL-18 and NLRP3 is also increased. Caspase 1 inhibitors can also reduce the body damage of mice by inhibiting the replication of CVB3 and activation of caspase 1. These results indicated that EV71 and CVB3 infections were associated with pyroptosis. In addition, Lei et al. found that [65], EV71 infection decreased the expression of GSDMD, the specific mechanism is that EV71 virus protease 3C cleaves GSDMD at Q193-G194 and inhibits the occurrence of pyroptosis, generating a non-functional GSDMD fragment composed of GSDMD1-193. The GSDMD-N fragment composed of GSDMD1-275 generated by caspase 1 cleavage can cause pyroptosis and thus inhibit the replication of EV71. These results suggest that EV71 virus can use its own $3 \mathrm{C}$ protease to escape the antiviral immune mechanism of the host (Table 1, Line 6).

\subsection{Role of Pyroptosis in Other Viral Infections}

Koraka et al. infected mice with rabies virus and found that the expressions of caspase 1, IL- $1 \beta$ and IL-18, which are closely related to pyroptosis, were significantly upregulated, indicating that rabies virus infection can activate pyroptosis signaling pathway [66].

A study of mouse rotavirus showed that GSDMD knockout mice were more susceptible to rotavirus and that caspase 1/11, GSDMD, and NLRP9b knockout tissues had greater rotavirus replication. In addition, they found that NLRP9b and the adaptor proteins ASC and caspase 1 assemble the inflammasome complex, which in turn mediates the pyroptosis of intestinal epithelial cells to inhibit rotavirus replication [67] (Table 1, Line 7).

In addition, studies have found that pyroptosis is also involved in the occurrence of many bacterial infectious diseases, such as Shigella frederi, Salmonella, Listeria, Yersinia and other bacteria can induce macrophage pyroptosis through the caspase 4/5/11-GSDMD pathway[35] [68].

\section{Conclusions and Perspectives}

In summary, pyroptosis plays important roles in the pathogenesis of many viral infectious diseases. Current studies on viral infectious diseases associated with pyroptosis are all found to occur through the caspase 1-GSDMD pathway, so we can directly inhibit the occurrence of pyroptosis by using the specific inhibitor of caspase 1 or inhibiting GSDMD to block the process of pyroptosis and reduce the expression levels of inflammatory cytokines IL- $1 \beta$ and IL-18, thus achieving the therapeutic effect of viral diseases, but the inhibitor should have side effects on the body. So it is still clear how to measure the use of drugs or inhibitors clinically. In addition, different viruses activate pyroptosis in different ways, such as receptors, inflammasomes and other related factors that regulate pyrop- 
tosis, so we can also deal with different targets.

GSDMD and GSDME are the key molecules in pyroptosis. It has been only found that GSDMD plays an important role in viral diseases, but whether GSDME is also involved in the vocalization and development of viral diseases remains to be explored. In addition, in addition to GSDMD and GSDME, whether there are other key molecules of pyroptosis and the specific mechanism of pyroptosis still need to be further studied. It's worth noting that cell pyroptosis can be used as a "double-edged sword" in viral infectious diseases. On the one hand, it helps to remove pathogens and prevent infection, which plays an indispensable role in maintaining the normal operation of the body. On the other hand, excessive pyroptosis can lead to intense inflammatory response, which aggravates the occurrence and development of the disease. As a newly discovered proinflammatory programmed cell death in recent years, pyroptosis will be proven to be involved in more and more viral diseases beyond the diseases summarized in this review. And due to the different stimulators of different viral infectious diseases, there may be a more complex mechanism to regulate the death process, which still needs to be further explored. Therefore, making an intensive study of the characteristics and mechanism of pyroptosis as well as its relationship with disease can provide a new way of thinking and effective drug targets for viral disease prevention and treatment.

\section{Conflicts of Interest}

The authors declare no conflicts of interest regarding the publication of this paper.

\section{References}

[1] Zychlinsky, A., Prevost, M.C. and Sansonetti, P.J. (1992) Shigella flexneri Induces Apoptosis in Infected Macrophages. Nature, 358, 167-169. https://doi.org/10.1038/358167a0

[2] Cookson, B.T. and Brennan, M.A. (2001) Pro-Inflammatory Programmed Cell Death. Trends in Microbiology, 9, 113-114. https://doi.org/10.1016/S0966-842X(00)01936-3

[3] Sborgi, L., Rühl, S., Mulvihill, E., Pipercevic, J., Heilig, R., Stahlberg, H., et al. (2016) GSDMD Membrane Pore Formation Constitutes the Mechanism of Pyroptotic Cell Death. The EMBO Journal, 35, 1766-1778. https://doi.org/10.15252/embj.201694696

[4] Ramos-Junior, E.S. and Morandini, A.C. (2017) Gasdermin: A New Player to the Inflammasome Game. Biomedical Journal, 40, 313-316. https://doi.org/10.1016/j.bj.2017.10.002

[5] Aglietti, R.A., Estevez, A., Gupta, A., Ramirez, M.G., Liu, P.S., Kayagaki, N., et al. (2016) GsdmD p30 Elicited by Caspase-11 during Pyroptosis Forms Pores in Membranes. Proceedings of the National Academy of Sciences of the United States of America, 113, 7858-7863. https://doi.org/10.1073/pnas.1607769113

[6] Khanova, E., Wu, R., Wang, W., Yan, R., Chen, Y., French, S.W., et al. (2018) Pyroptosis by caspase11/4-gasdermin-D Pathway in Alcoholic Hepatitis in Mice and 
Patients. Hepatology, 67, 1737-1753. https://doi.org/10.1002/hep.29645

[7] Liu, X., Zhang, Z., Ruan, J., Pan, Y., Magupalli, V.G., Wu, H., et al. (2016) Inflammasome-Activated Gasdermin D Causes Pyroptosis by Forming Membrane Pores. Nature, 535, 153-158. https://doi.org/10.1038/nature18629

[8] Mulvihill, E., Sborgi, L., Mari, S.A., Pfreundschuh, M., Hiller, S. and Müller, D.J. (2018) Mechanism of Membrane Pore Formation by Human Gasdermin-D. The EMBO Journal, 37, e98321. https://doi.org/10.15252/embj.201798321

[9] Saeki, N., Usui, T., Aoyagi, K., Kim, D.H., Sato, M., Mabuchi, T., et al. (2009) Distinctive Expression and Function of Four GSDM Family Genes (GSDMA-D) in Normal and Malignant Upper Gastrointestinal Epithelium. Genes Chromosomes Cancer, 48, 261-271. https://doi.org/10.1002/gcc.20636

[10] Shi, J., Gao, W. and Shao, F. (2017) Pyroptosis: Gasdermin-Mediated Programmed Necrotic Cell Death. Trends in Biochemical Sciences, 42, 245-254. https://doi.org/10.1016/j.tibs.2016.10.004

[11] Tsuchiya, K., Nakajima, S., Hosojima, S., Thi Nguyen, D., Hattori, T., Manh Le, T., et al. (2019) Caspase-1 Initiates Apoptosis in the Absence of Gasdermin D. Nature Communications, 10, 2091. https://doi.org/10.1038/s41467-019-09753-2

[12] McIlwain, D.R., Berger, T. and Mak, T.W. (2015) Caspase Functions in Cell Death and Disease. Cold Spring Harbor Perspectives in Biology, 5, a008656. https://doi.org/10.1101/cshperspect.a026716

[13] Yazdi, A.S., Guarda, G., D’Ombrain, M.C. and Drexler, S.K. (2010) Inflammatory Caspases in Innate Immunity and Inflammation. Journal of Innate Immunity, 2, 228-237. https://doi.org/10.1159/000283688

[14] Angosto, D., López-Castejón, G., López-Muñoz, A., Sepulcre, M.P., Arizcun, M., Meseguer, J., et al. (2012) Evolution of Inflammasome Functions in Vertebrates: Inflammasome and Caspase-1 Trigger Fish Macrophage Cell Death But Are Dispensable for the Processing of IL-1 $\beta$. Innate Immunity, 18, 815-824.

https://doi.org/10.1177/1753425912441956

[15] Kumar, S., White, D.L., Takai, S., Turczynowicz, S., Juttner, C.A. and Hughes, T.P. (1995) Apoptosis Regulatory Gene NEDD2 Maps to Human Chromosome Segment 7q34-35, a Region Frequently Affected in Haematological Neoplasms. Human Genetics, 95, 641-644. https://doi.org/10.1007/BF00209480

[16] Li, P., Zhou, L., Zhao, T., Liu, X., Zhang, P., Liu, Y., et al. (2017) Caspase-9: Structure, Mechanisms and Clinical Application. Oncotarget, 8, 23996-24008. https://doi.org/10.18632/oncotarget.15098

[17] Orning, P., Weng, D., Starheim, K., Ratner, D., Best, Z., Lee, B., et al. (2018) Pathogen Blockade of TAK1 Triggers Caspase-8-Dependent Cleavage of Gasdermin D and Cell Death. Science, 362, 1064-1069. https://doi.org/10.1126/science.aau2818

[18] Shalini, S., Dorstyn, L., Dawar, S. and Kumar, S. (2015) Old, New and Emerging Functions of Caspases. Cell Death \& Differentiation, 22, 526-539. https://doi.org/10.1038/cdd.2014.216

[19] Chen, S., Mei, S., Luo, Y., Wu, H., Zhang, J. and Zhu, J. (2018) Gasdermin Family: A Promising Therapeutic Target for Stroke. Translational Stroke Research, 9, 555-563. https://doi.org/10.1007/s12975-018-0666-3

[20] Jorgensen, I., Rayamajhi, M. and Miao, E.A. (2017) Programmed Cell Death as a Defence against Infection. Nature Reviews Immunology, 17, 151-164.

https://doi.org/10.1038/nri.2016.147

[21] Chao, K.L., Kulakova, L. and Herzberg, O. (2017) Gene Polymorphism Linked to 
Increased Asthma and IBD Risk Alters Gasdermin-B Structure, a Sulfatide and Phosphoinositide Binding Protein. Proceedings of the National Academy of Sciences of the United States of America, 114, e1128-e1137. https://doi.org/10.1073/pnas.1616783114

[22] Das, S., Miller, M. and Broide, D.H. (2017) Chromosome 17q21 Genes ORMDL3 and GSDMB in Asthma and Immune Diseases. Advances in Immunology, 135, 1-52. https://doi.org/10.1016/bs.ai.2017.06.001

[23] Kovacs, S.B. and Miao, E.A. (2017) Gasdermins: Effectors of Pyroptosis. Trends in Cell Biology, 27, 673-684. https://doi.org/10.1016/j.tcb.2017.05.005

[24] Saeki, N., Kuwahara, Y., Sasaki, H., Satoh, H. and Shiroishi, T. (2000) Gasdermin (Gsdm) Localizing to Mouse Chromosome 11 Is Predominantly Expressed in Upper Gastrointestinal Tract But Significantly Suppressed in Human Gastric Cancer Cells. Mammalian Genome, 11, 718-724. https://doi.org/10.1007/s003350010138

[25] Feng, S., Fox, D. and Man, S.M. (2018) Mechanisms of Gasdermin Family Members in Inflammasome Signaling and Cell Death. Journal of Molecular Biology, 430, 3068-3080. https://doi.org/10.1016/j.jmb.2018.07.002

[26] Ding, J., Wang, K., Liu, W., She, Y., Sun, Q., Shi, J., et al. (2016) Pore-Forming Activity and Structural Autoinhibition of the Gasdermin Family. Nature, 535, 111-116. https://doi.org/10.1038/nature18590

[27] Liu, Z., Wang, C., Rathkey, J.K., Yang, J., Dubyak, G.R., Abbott, D.W., et al. (2018) Structures of the Gasdermin D C-Terminal Domains Reveal Mechanisms of Autoinhibition. Structure, 26, 778-784.e3. https://doi.org/10.1016/j.str.2018.03.002

[28] Wang, Y., Yin, B., Li, D., Wang, G., Han, X. and Sun, X. (2018) GSDME Mediates Caspase-3-Dependent Pyroptosis in Gastric Cancer. Biochemical and Biophysical Research Communications, 495, 1418-1425. https://doi.org/10.1016/j.bbrc.2017.11.156

[29] Rogers, C., Fernandes-Alnemri, T., Mayes, L., Alnemri, D., Cingolani, G. and Alnemri, E.S. (2017) Cleavage of DFNA5 by Caspase-3 during Apoptosis Mediates Progression to Secondary Necrotic/Pyroptotic Cell Death. Nature Communications, 8, Article No. 14128. https://doi.org/10.1038/ncomms14128

[30] Wang, Y., Gao, W., Shi, X., Ding, J., Liu, W., He, H., et al. (2017) Chemotherapy Drugs Induce Pyroptosis through Caspase-3 Cleavage of a Gasdermin. Nature, 547, 99-103. https://doi.org/10.1038/nature22393

[31] Keller, M., Rüegg, A., Werner, S. and Beer, H.D. (2008) Active Caspase-1 Is a Regulator of Unconventional Protein Secretion. Cell, 132, 818-831. https://doi.org/10.1016/j.cell.2007.12.040

[32] Sharma, D. and Kanneganti, T.D. (2016) The Cell Biology of Inflammasomes: Mechanisms of Inflammasome Activation and Regulation. Journal of Cell Biology, 213, 617-629. https://doi.org/10.1083/jcb.201602089

[33] Faustin, B., Lartigue, L., Bruey, J.M., Luciano, F., Sergienko, E., Bailly-Maitre, B., et al. (2007) Reconstituted NALP1 Inflammasome Reveals Two-Step Mechanism of Caspase-1 Activation. Molecular Cell, 25, 713-724. https://doi.org/10.1016/j.molcel.2007.01.032

[34] Broz, P. (2015) Immunology: Caspase Target Drives Pyroptosis. Nature, 526, 642-643. https://doi.org/10.1038/nature15632

[35] Vanaja, S.K., Russo, A.J., Behl, B., Banerjee, I., Yankova, M., Deshmukh, S.D., et al. (2016) Bacterial Outer Membrane Vesicles Mediate Cytosolic Localization of LPS and Caspase-11 Activation. Cell, 165, 1106-1119. https://doi.org/10.1016/j.cell.2016.04.015 
[36] Taylor, R.C., Cullen, S.P. and Martin, S.J. (2008) Apoptosis: Controlled Demolition at the Cellular Level. Nature Reviews Molecular Cell Biology, 9, 231-241. https://doi.org/10.1038/nrm2312

[37] Tait, S.W. and Green, D.R. (2010) Mitochondria and Cell Death: Outer Membrane Permeabilization and Beyond. Nature Reviews Molecular Cell Biology, 11, 621-632. https://doi.org/10.1038/nrm2952

[38] Coronaviridae Study Group of the International Committee on Taxonomy of Viruses (2020) The Species Severe Acute Respiratory Syndrome-Related Coronavirus: Classifying 2019-nCoV and Naming It SARS-CoV-2. Nature Microbiology, 5, 536-544. https://doi.org/10.1038/s41564-020-0695-Z

[39] Zhu, N., Zhang, D., Wang, W., Li, X., Yang, B., Song, J., et al. (2020) A Novel Coronavirus from Patients with Pneumonia in China, 2019. The New England Journal of Medicine, 382, 727-733. https://doi.org/10.1056/NEJMoa2001017

[40] Chen, G., Wu, D., Guo, W., Cao, Y., Huang, D., Wang, H., et al. (2020) Clinical and Immunological Features of Severe and Moderate Coronavirus Disease 2019. Journal of Clinical Investigation, 130, 2620-2629. https://doi.org/10.1172/JCI137244

[41] Soy, M., Keser, G., Atagündüz, P., Tabak, F., Atagündüz, I. and Kayhan, S. (2020) Cytokine Storm in COVID-19: Pathogenesis and Overview of Anti-Inflammatory Agents Used in Treatment. Clinical Rheumatology, 39, 2085-2094. https://doi.org/10.1007/s10067-020-05190-5

[42] Ratajczak, M.Z., Bujko, K., Ciechanowicz, A., Sielatycka, K., Cymer, M., Marlicz, W., et al. (2021) SARS-CoV-2 Entry Receptor ACE2 Is Expressed on Very Small CD45(-) Precursors of Hematopoietic and Endothelial Cells and in Response to Virus Spike Protein Activates the Nlrp3 Inflammasome. Stem Cell Reviews and Reports, 17, 266-277. https://doi.org/10.1007/s12015-020-10010-Z

[43] Ratajczak, M.Z. and Kucia, M. (2020) SARS-CoV-2 Infection and Overactivation of Nlrp3 Inflammasome as a Trigger of Cytokine "Storm" and Risk Factor for Damage of Hematopoietic Stem Cells. Leukemia, 34, 1726-1729. https://doi.org/10.1038/s41375-020-0887-9

[44] Freeman, T.L. and Swartz, T.H. (2020) Targeting the NLRP3 Inflammasome in Severe COVID-19. Frontiers in Immunology, 11, 1518. https://doi.org/10.3389/fimmu.2020.01518

[45] Franchi, L., Muñoz-Planillo, R. and Núñez, G. (2012) Sensing and Reacting to Microbes through the Inflammasomes. Nature Immunology, 13, 325-332. https://doi.org/10.1038/ni.2231

[46] Ratajczak, M.Z., Bujko, K., Cymer, M., Thapa, A., Adamiak, M., Ratajczak, J., et al. (2020) The Nlrp3 Inflammasome as a "Rising Star" in Studies of Normal and Malignant Hematopoiesis. Leukemia, 34, 1512-1523. https://doi.org/10.1038/s41375-020-0827-8

[47] Swanson, K.V., Deng, M. and Ting, J.P. (2019) The NLRP3 Inflammasome: Molecular Activation and Regulation to Therapeutics. Nature Reviews Immunology, 19, 477-489. https://doi.org/10.1038/s41577-019-0165-0

[48] Zheng, Y., Huang, Z., Yin, G., Zhang, X., Ye, W., Hu, Z., et al. (2020) Study of the Lymphocyte Change between COVID-19 and Non-COVID-19 Pneumonia Cases Suggesting Other Factors besides Uncontrolled Inflammation Contributed to Multi-Organ Injury. https://doi.org/10.2139/ssrn.3555267

[49] Cao, M., Zhang, D., Wang, Y., Lu, Y., Zhu, X., Li, Y., et al. (2020) Clinical Features of Patients Infected with the 2019 Novel Coronavirus (COVID-19) in Shanghai, China. https://doi.org/10.1101/2020.03.04.20030395 
[50] Kuiken, T., Riteau, B., Fouchier, R.A. and Rimmelzwaan, G.F. (2012) Pathogenesis of Influenza Virus Infections: The Good, the Bad and the Ugly. Current Opinion in Virology, 2, 276-286. https://doi.org/10.1016/j.coviro.2012.02.013

[51] Chen, Y.J., Wang, S.F., Weng, I.C., Hong, M.H., Lo, T.H., Jan, J.T., et al. (2018) Galectin-3 Enhances Avian H5N1 Influenza A Virus-Induced Pulmonary Inflammation by Promoting NLRP3 Inflammasome Activation. The American Journal of Pathology, 188, 1031-1042. https://doi.org/10.1016/j.ajpath.2017.12.014

[52] Kuriakose, T., Man, S.M., Malireddi, R.K., Karki, R., Kesavardhana, S., Place, D.E., et al. (2016) ZBP1/DAI Is an Innate Sensor of Influenza Virus Triggering the NLRP3 Inflammasome and Programmed Cell Death Pathways. Science Immunology, 1, aag2045. https://doi.org/10.1126/sciimmunol.aag2045

[53] Fanales-Belasio, E., Raimondo, M., Suligoi, B. and Buttò, S. (2010) HIV Virology and Pathogenetic Mechanisms of Infection: A Brief Overview. Annali dell Istituto Superiore di Sanità, 46, 5-14. https://doi.org/10.1590/S0021-25712010000100002

[54] Vidya Vijayan, K.K., Karthigeyan, K.P., Tripathi, S.P. and Hanna, L.E. (2017) Pathophysiology of CD4+ T-Cell Depletion in HIV-1 and HIV-2 Infections. Frontiers in Immunology, 8, 580. https://doi.org/10.3389/fimmu.2017.00580

[55] Church, J.A. (2014) Cell Death by Pyroptosis Drives CD4 T-Cell Depletion in HIV-1 Infection. Pediatrics, 134, S184. https://doi.org/10.1542/peds.2014-1817J

[56] Doitsh, G. and Greene, W.C. (2016) Dissecting How CD4 T Cells Are Lost during HIV Infection. Cell Host \& Microbe, 19, 280-291.

https://doi.org/10.1016/j.chom.2016.02.012

[57] Wree, A., Eguchi, A., McGeough, M.D., Pena, C.A., Johnson, C.D., Canbay, A., et al. (2014) NLRP3 Inflammasome Activation Results in Hepatocyte Pyroptosis, Liver Inflammation, and Fibrosis in Mice. Hepatology, 59, 898-910. https://doi.org/10.1002/hep.26592

[58] Tacke, F. (2017) Targeting Hepatic Macrophages to Treat Liver Diseases. Journal of Hepatology, 66, 1300-1312. https://doi.org/10.1016/j.jhep.2017.02.026

[59] Yu, X., Lan, P., Hou, X., Han, Q., Lu, N., Li, T., et al. (2017) HBV Inhibits LPS-Induced NLRP3 Inflammasome Activation and IL- $1 \beta$ Production via Suppressing the NF- $\kappa$ B Pathway and ROS Production. Journal of Hepatology, 66, 693-702. https://doi.org/10.1016/j.jhep.2016.12.018

[60] Kofahi, H.M., Taylor, N.G., Hirasawa, K., Grant, M.D. and Russell, R.S. (2016) Hepatitis C Virus Infection of Cultured Human Hepatoma Cells Causes Apoptosis and Pyroptosis in Both Infected and Bystander Cells. Scientific Reports, 6, Article No. 37433. https://doi.org/10.1038/srep37433

[61] Wang, J., Ren, H., Yuan, X., Ma, H., Shi, X. and Ding, Y. (2018) Interleukin-10 Secreted by Mesenchymal Stem Cells Attenuates Acute Liver Failure through Inhibiting Pyroptosis. Hepatology Research, 48, e194-e202.

https://doi.org/10.1111/hepr.12969

[62] Tan, T.Y. and Chu, J.J.H. (2013) Dengue Virus-Infected Human Monocytes Trigger Late Activation of Caspase-1, Which Mediates Pro-Inflammatory IL- $1 \beta$ Secretion and Pyroptosis. Journal of General Virology, 94, 2215-2220. https://doi.org/10.1099/vir.0.055277-0

[63] Wu, M.F., Chen, S.T., Yang, A.H., Lin, W.W., Lin, Y.L., Chen, N.J., et al. (2013) CLEC5A Is Critical for Dengue Virus-Induced Inflammasome Activation in Human Macrophages. Blood, 121, 95-106. https://doi.org/10.1182/blood-2012-05-430090

[64] Wang, Y., Qin, Y., Wang, T., Chen, Y., Lang, X., Zheng, J., et al. (2018) Pyroptosis Induced by Enterovirus 71 and Coxsackievirus B3 Infection Affects Viral Replica- 
tion and Host Response. Scientific Reports, 8, Article No. 2887. https://doi.org/10.1038/s41598-018-20958-1

[65] Lei, X., Zhang, Z., Xiao, X., Qi, J., He, B. and Wang, J. (2017) Enterovirus 71 Inhibits Pyroptosis through Cleavage of Gasdermin D. Journal of Virology, 91, 17. https://doi.org/10.1128/JVI.01069-17

[66] Koraka, P., Martina, B.E.E., Smreczak, M., Orlowska, A., Marzec, A., Trebas, P., et al. (2019) Inhibition of Caspase-1 Prolongs Survival of Mice Infected with Rabies Virus. Vaccine, 37, 4681-4685. https://doi.org/10.1016/j.vaccine.2018.04.002

[67] Zhu, S., Ding, S., Wang, P., Wei, Z., Pan, W., Palm, N.W., et al. (2017) Nlrp9b Inflammasome Restricts Rotavirus Infection in Intestinal Epithelial Cells. Nature, 546, 667-670. https://doi.org/10.1038/nature22967

[68] Maltez, V.I., Tubbs, A.L., Cook, K.D., Aachoui, Y., Falcone, E.L., Holland, S.M., et al. (2015) Inflammasomes Coordinate Pyroptosis and Natural Killer Cell Cytotoxicity to Clear Infection by a Ubiquitous Environmental Bacterium. Immunity, 43, 987-997. https://doi.org/10.1016/j.immuni.2015.10.010 